\title{
Tuning of energy levels and optical properties of graphene quantum dots
}

\author{
Z. Z. Zhang and Kai Chang* \\ SKLSM, Institute of Semiconductors, Chinese Academy of Sciences, P.O. Box 912, Beijing 100083, People's Republic of China \\ F. M. Peeters \\ Departement Fysica, Universiteit Antwerpen, Groenenborgerlaan 171, B-2020 Antwerpen, Belgium
}

(Received 25 February 2008; revised manuscript received 4 May 2008; published 6 June 2008)

\begin{abstract}
We investigate theoretically the magnetic levels and optical properties of zigzag- and armchair-edged hexagonal graphene quantum dots (GQDs) utilizing the tight-binding method. A bound edge state at zero energy appears for the zigzag GQDs in the absence of a magnetic field. The magnetic levels of GQDs exhibit a Hofstadter-butterfly spectrum and approach the Landau levels of two-dimensional graphene as the magnetic field increases. The optical properties are tuned by the size, the type of the edge, and the external magnetic field.
\end{abstract}

DOI: $10.1103 /$ PhysRevB.77.235411

PACS number(s): 73.22.-f, 78.67.-n, 75.75.+a, 81.07.Nb

Graphene is a single atomic layer consisting of a twodimensional honeycomb lattice of carbon atoms. This system has attracted intense attention because of fundamental physics and promising applications in nanoelectronics. ${ }^{1,2}$ It exhibits high crystal quality, an exotic Dirac-type spectrum, and ballistic transport properties on a submicron scale. Graphene samples are usually fabricated by micromechnical cleavage of graphite and have excellent mechanical properties that make it possible to sustain huge electric currents. The lateral confinement of Dirac fermions in graphene is still an enigmatic and extremely challenging task due to the well-known Klein paradox. The Klein paradox makes it impossible to localize the carriers in a confined region utilizing an electrostatic gate. The confinement of Dirac fermions at a nanometer scale is one of the central goals of graphene-based electronics and has attracted increasing interest. ${ }^{3-8}$ Recently, it was demonstrated experimentally that graphene can be cut in the desired shape and size., ${ }^{1,2}$ Recent progresses in fabricating and characterizing stable graphene nanostructures provides the opportunity to explore the various remarkable optical $^{9-11}$ and transport properties ${ }^{12}$ of these structures.

In this work, we investigate theoretically the electronic structure and optical properties of zigzag- and armchairedged hexagonal graphene quantum dots (GQDs) (see Fig. 1) utilizing the nearest-neighbor tight-binding model. The dangling bonds at the edges are passivated by hydrogen atoms. The model has been successfully used for fullerene molecules, carbon nanotubes, and other carbon-related materials. ${ }^{13-16}$ The Hamiltonian of GQDs can be written as $H=\sum_{i} \varepsilon_{i} c_{i}^{\dagger} c_{i}+\sum_{\langle i, j\rangle} t_{i, j} c_{i}^{\dagger} c_{j}$, where $\varepsilon_{i}$ is the site energy, $t_{i j}$ is the transfer energy between the nearest-neighbor sites, and $c_{i}^{\dagger}$ $\left(c_{i}\right)$ is the creation (annihilation) operator of the $\pi$ electron at the site $i$. When considering a magnetic field $B$ applied perpendicularly to the plane of a GQD, the transfer integral $t_{i j}$ becomes $t_{i j}=t e^{i 2 \pi \phi_{i, j}}$, where $\phi_{i j}=\frac{e}{h} \int_{r_{i}}^{r_{j}} d \mathbf{l} \cdot \mathbf{A}$ is the Peierls phase. $\mathbf{A}=(0, B x, 0)$ is the vector potential corresponding to the magnetic field $B$ along the $z$ axis, which is perpendicular to the graphene plane. In our calculation, we take $\Phi_{0}=h / e$ as the unit of the magnetic flux and $\Phi=\sqrt{3} B a_{0}^{2} / 2$ as the magnetic flux through a plaquette, where $a_{0}=2.46 \AA$ is the lattice constant of graphite. The difference between the values of $\varepsilon_{i}$ and $t_{i j}$ for the atoms at the edge and the center is neglected. The relevant parameters used in our calculation are $\varepsilon=0$ and $t=-3.033$. ${ }^{15}$ The eigenvalues and eigenstates can be obtained from the secular equation $\operatorname{det}|\varepsilon-H|=0$, where $H_{i i}=0, H_{\langle i, j\rangle}=t e^{i 2 \pi \phi_{i, j}}$.

Figure 1 shows the electronic density distributions of the zigzag and armchair-edged graphene quantum dots (ZGQD and AGQD, respectively), in the absence of a magnetic field. The size of a dot is characterized by $N$, the number of hexagonal units along an edge. Figures 1(a) and 1(b) show the probability distributions of the highest valence level (HVL) and the lowest conduction level (LCL) for the ZGQD with small size $(N=2)$. The probability distributions of the HVL
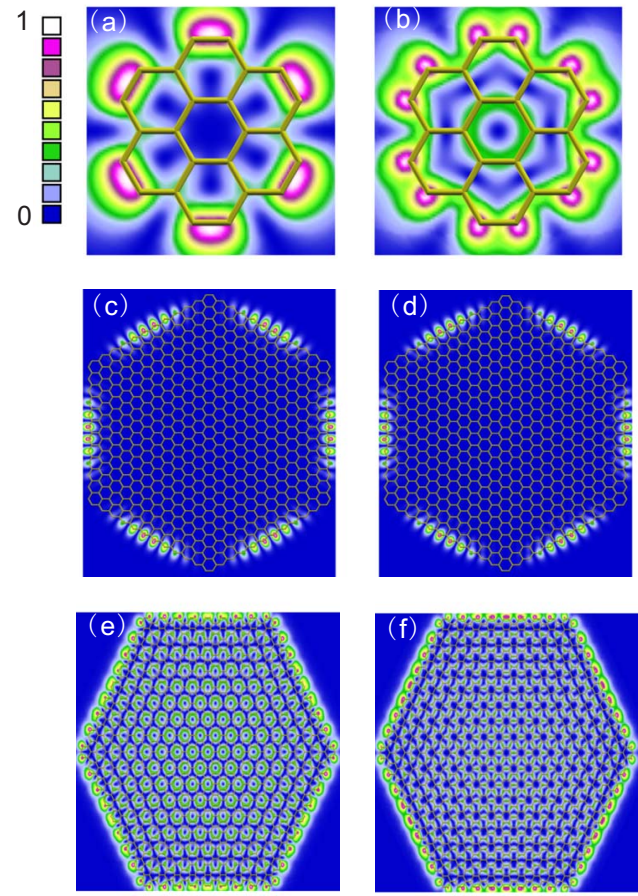

FIG. 1. (Color online) Electronic density distributions of the HVL and LCL in the absence of a magnetic field. Panels (a) and (b): HVL and LCL for the $N_{z}=2$ ZGQD. Panels (c) and (d): the same with $N_{z}=12$. Panels (e) and (f): the same with AGQD, but $N_{A}=9$. 
(a)

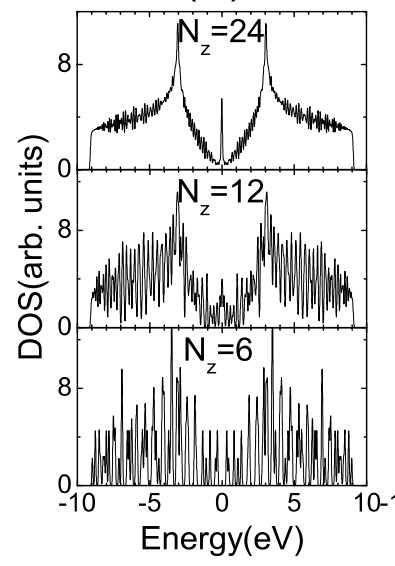

(b)

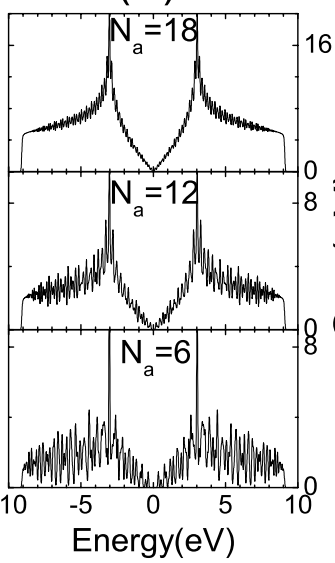

(c)

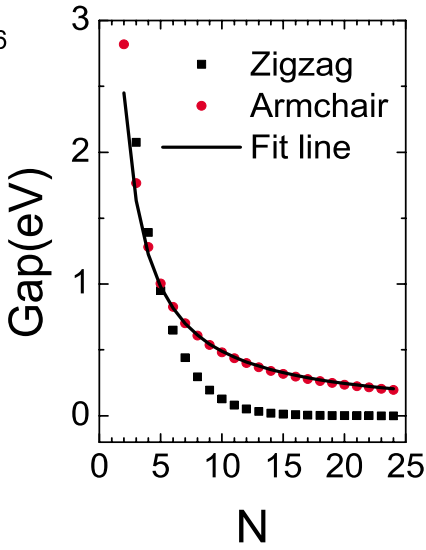

FIG. 2. (Color online) Density of states of (a) ZGQD and (b) AGQD. We use a Gaussian function $f(E)=e^{-\left(E-E_{0}\right)^{2} / \Gamma^{2}}$ with a broadening factor $\Gamma=0.05 \mathrm{eV}$ to smooth the discontinuous energy spectra. (c) The gap of ZGQD and AGQD as a function of the size, and the function of the fit line is $a / N$ with $a=4.9 \mathrm{eV}$. and LCL correspond to the bonding and antibonding states that are localized at the corner of the hexagonal GQD. In contrast to conventional semiconductor quantum dots where the ground state is localized at the center of the dot, the ground states for the conduction and valence bands, i.e., HVL and LCL, localize at the middle of each edge in the ZGQD [see Figs. 1(c) and 1(d)] as the size of the ZGQD increases. This feature can be understood as follows: the Dirac fermion in a ZGQD behaves like a confined photon in a cavity and the lowest mode is the whispering gallery mode, which also localizes at the boundary of the cavity. The difference between the bonding and antibonding states becomes smaller as the size of the ZGQD increases. The difference between the edge states of a ZGQD and a graphene nanoribbon is that the edge state of the ZGQD localizes at the middle of the edge of GQD, in contrast to the homogeneously distributed edge state of a zigzag graphene nanoribbon ${ }^{14,17}$ or a zigzag triangular GQD. ${ }^{18}$ This occurs because the contribution of each carbon atom at the edge of a ZGQD to the edge state is different, while it is the same for a zigzag nanoribbon or a zigzag triangular GQD. The density distributions of the LCL and HVL in an AGQD [see Figs. 1(e) and 1(f)] extend more completely over the whole GQD region and are very different from that in a ZGQD. This difference is indeed caused by the different topological geometry of the boundary of the graphene nanostructures.

Figures 2(a) and 2(b) show the density of states (DOS) of ZGQDs and AGQDs, respectively, with different sizes in the absence of a magnetic field. The total number of the carbon atoms in ZGQD and AGQD are $6 N_{z}^{2}$ and $6\left(3 N_{a}^{2}-3 N_{a}+1\right)$, respectively. From the figures, we find that there is no edge state in a small ZGQD and the edge state appears when the size of the ZGQD increases according to the states at zero energy. Meanwhile, there is never an edge state for the AGQD. To demonstrate how the edge state appears, we plot the energy gap, i.e., the energy difference between the LCL and the HVL, as a function of the size $(N)$ of the GQD in Fig. 2(c). The energy gap decreases as the size of the GQD increases. Interestingly, the energy gap of the zigzag (armchair) GQD decays to zero quickly (slowly) as the size of the GQD increases. When the size of the AGQD approaches infinity, the gap decreases to zero, i.e., we recover the twodimensional graphene case. The calculated energy gap for

the AGQD falls off as $1 / N \propto 1 / L$ [see the solid line in Fig. 2(c)], where $L$ is the length of each edge of the hexagonal GQD. This dependence of the band gap on the size of GQD is very different from that of a conventional semiconductor quantum dot, which behaves as $1 / L^{2}$.

Figures 3(a) and 4(a) depict the magnetic field dependence of the energy spectrum of a ZGQD and an AGQD exhibiting a clear Hofstadter-butterfly characteristic, which is fractal and exhibits self-similarity. ${ }^{14,19-21}$ As the magnetic flux increases, the magnetic levels in the GQD, i.e., the socalled Fock--Darwin levels, approach the Landau levels [see

(a)

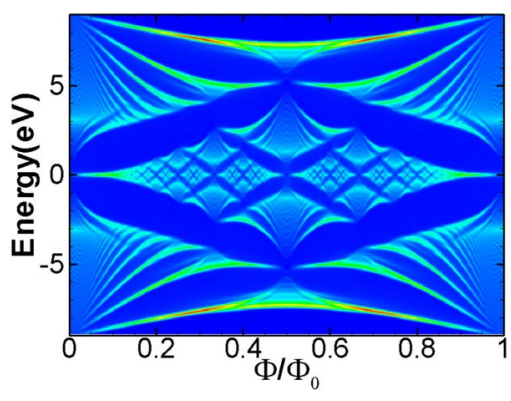

(d)

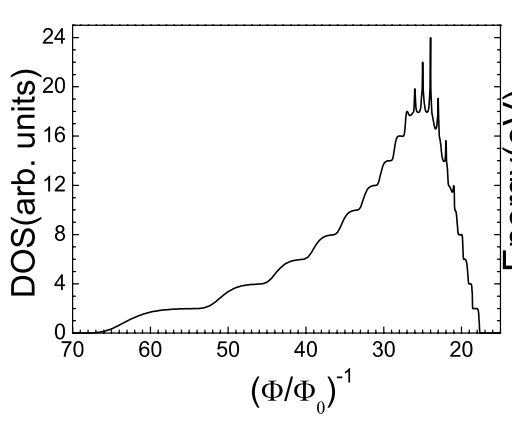

FIG. 3. (Color online) (a) The DOS and energy spectrum of the $N_{z}=12$ ZGQD in a magnetic field. We use a Gauss function with a broadening factor of $0.1 \mathrm{eV}$ to smoothen the discontinuous energy spectra. [(b) and (c)] The magnetic energy level fan near the Dirac point, i.e., the zero energy point. The red lines in (b) correspond to the Landau level of two-dimensional graphene. (d) the DOS at the Dirac point, as a function of the inverse flux $\Phi / \Phi_{0}$, where we use a Gauss function with a small broadening factor of $0.01 \mathrm{meV}$. 
(a)

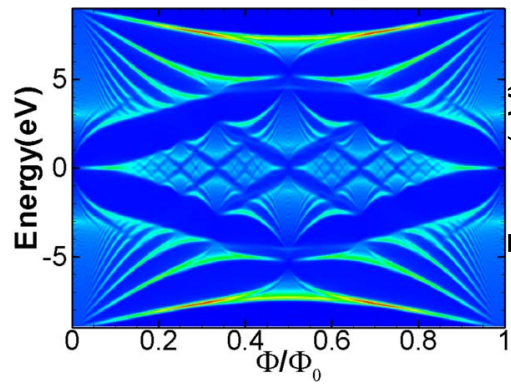

(d)
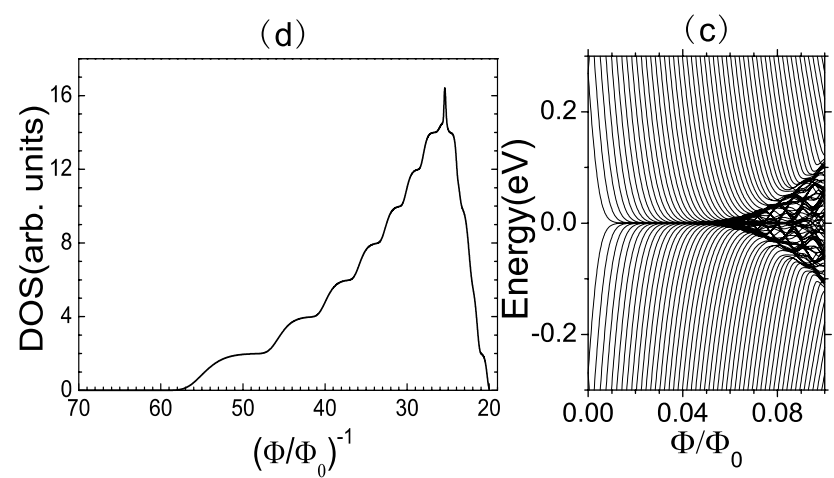

FIG. 4. (Color online) The same as Fig. 3, but for the $N_{a}=9$ AGQD

the red lines in Figs. 3(b) and $4(\mathrm{~b})]$ in graphene $E_{n}$ $=\operatorname{sgn}(n)\left(\sqrt{3} t a_{0} / 2 l_{B}\right) \sqrt{2|n|}$, where $l_{B}=\sqrt{\hbar / e B}$ is the cyclotron radius, $n$ is an integer, and sgn is the sign function.

Figures 3(c) and 4(c) show in detail how the magnetic levels of ZGQD and AGQD approach the zeroth Landau level at small magnetic flux. As the magnetic flux increases, more energy levels approach the zeroth Landau level in pairs. The degeneracy of the energy level at zero energy will reach its maximum value $2 N$ for $\Phi / \Phi_{0}=1 / 2 N$. When the magnetic flux increases further, the degeneracy of the energy level at zero energy is lifted fast. This feature can also be seen in Fig. 3(d), which plots the DOS at the Dirac point. This figure indicates that the degeneracy, i.e., the number of energy levels at the zero energy, approximately decreases inverse linearly with the magnetic flux $\Phi / \Phi_{0}$. These figures clearly demonstrate that the energy spectrum of the GQD possesses electron-hole symmetry when we neglect the second-nearest-neighbor interaction. The DOS and the magnetic level fan of the AGQD are similar to that of the ZGQD except at small magnetic flux. Comparing Fig. 3(c) to Fig. 4(c), the magnetic levels in the AGQD are distinct from those in the ZGQD at small magnetic flux because the ZGQD shows the edge state and AGQD does not for the levels near the Dirac point in the absence of the magnetic field. Therefore, the magnetic levels exhibit distinct behavior as the magnetic flux increases. The DOS of the AGQD [see Fig. 4(d)] also shows a steplike feature as the magnetic flux at the Dirac point increases.

Figure 5 describes the density distributions of the LCL and HVL in the ZGQD and AGQD at small magnetic flux $\Phi / \Phi_{0}=0.01$. Interestingly, the density distributions of the LCL and HVL penetrate into the center of the GQD for the
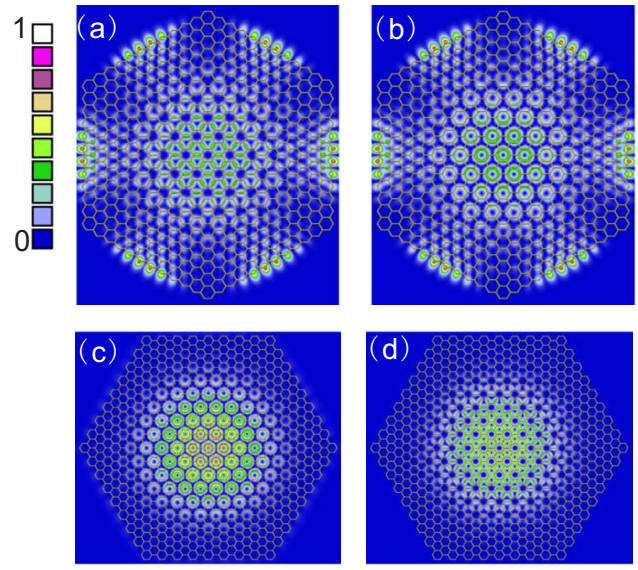

FIG. 5. (Color online) (a) and (b) show the density distributions of the HVL and LCL for the $N_{z}=12$ ZGQD in the presence of the magnetic flex $\Phi / \Phi_{0}=0.01$, respectively. (c) and (d) are the same as (a) and (b), but for the $N_{a}=9$ AGQD.

ZGQDs, which is very different from the AGQD case where both the electron and hole are dominantly localized in the center of the GQD. The density distributions for the ZGQD and AGQD show a $C_{6 v}$ symmetry. This characteristic is caused by the magnetic confinement when the magnetic length $l_{B}$ becomes comparable with the size of the GQD. In addition to those differences, the LCL and HVL of the zigzag GQD show an opposite symmetry order with respect to that of the armchair GQD, i.e., the LCL (HVL) and HVL (LCL) of the ZGQD (AGQD) belong to the $E_{1}\left(E_{2}\right)$ and $E_{2}\left(E_{1}\right)$ representations at zero magnetic field [see Fig. 6].

The optical properties of GQDs are promising for potential applications in optic-electronic devices based on graphene. Therefore, we calculate the absorption spectra of GQD $\alpha(\hbar \omega)=\frac{\pi e^{2}}{m_{0}^{2} \varepsilon_{0} c n \omega V} \sum_{c, v}\left|\vec{\varepsilon} \cdot P_{c v}\right|^{2} \times \delta\left(E_{c}-E_{v}-\hbar \omega\right)$, where $n$ is the refractive index, $c$ is the speed of light in vacuum, $\varepsilon_{0}$ is the permittivity of vacuum, $m_{0}$ is the free-electron mass, and $\vec{\varepsilon}$ is the polarization vector of the incident light along the $x$ direction. The coupling between the $s p_{2}$ states and the $p_{z}$ state is neglected since we are only interested in the optical properties of the GQD near the Dirac point, i.e., at the low energy regime. The momentum matrix ${ }^{22}$ is $\langle n|\mathbf{p}| m\rangle$ $=i m_{0} / \hbar \Sigma_{\mathbf{r}} \Sigma_{\mathbf{r}^{\prime}} c_{\mathbf{r}^{*}}^{*} c_{\mathbf{r}^{\prime}}\left(\mathbf{r}^{\prime}-\mathbf{r}\right)\left\langle p_{z}, \mathbf{r}|H| p_{z}, \mathbf{r}^{\prime}\right\rangle$. The momentum operator $p_{x}\left(p_{y}\right)$ has $E_{2}$ symmetry and its direct product with all the irreducible representations of the $\mathrm{C}_{6 v}$ group can be found in Table I. We divide the levels of the GQD into two different families: $A_{1}, A_{3}, E_{1} \in \Omega_{1}$ and $A_{2}, A_{4}, E_{2} \in \Omega_{2}$. The symmetry requires that only transitions between the valence and conduction band levels belonging to the different families $\Omega_{1}$ and $\Omega_{2}$ are allowed. Notice that the initial or final states of the transition should belong to the $E_{1}$ or $E_{2}$ representations. In Figs. 6(a) and 6(d), we label the level structure of a $N_{z}$ $=12$ and $N_{a}=9 \mathrm{GQD}$ near the Dirac point as $C_{1}-C_{n}$ for conduction bands with ascending order and $V_{1}-V_{n}$ for valence bands with descending order, respectively. The conduction $C_{i}$ and valence band levels $V_{i}$ belong to the distinct families $\Omega_{1}$ and $\Omega_{2}$, respectively. For example, if $C_{i}$ belongs to the family $\Omega_{1}$, i.e., $A_{1}, A_{3}$ or $E_{1}, V_{i}$ must belong to the family $\Omega_{2}$, i.e., $A_{2}, A_{4}$ or $E_{2}$, or vice versa. For zigzag GQDs 

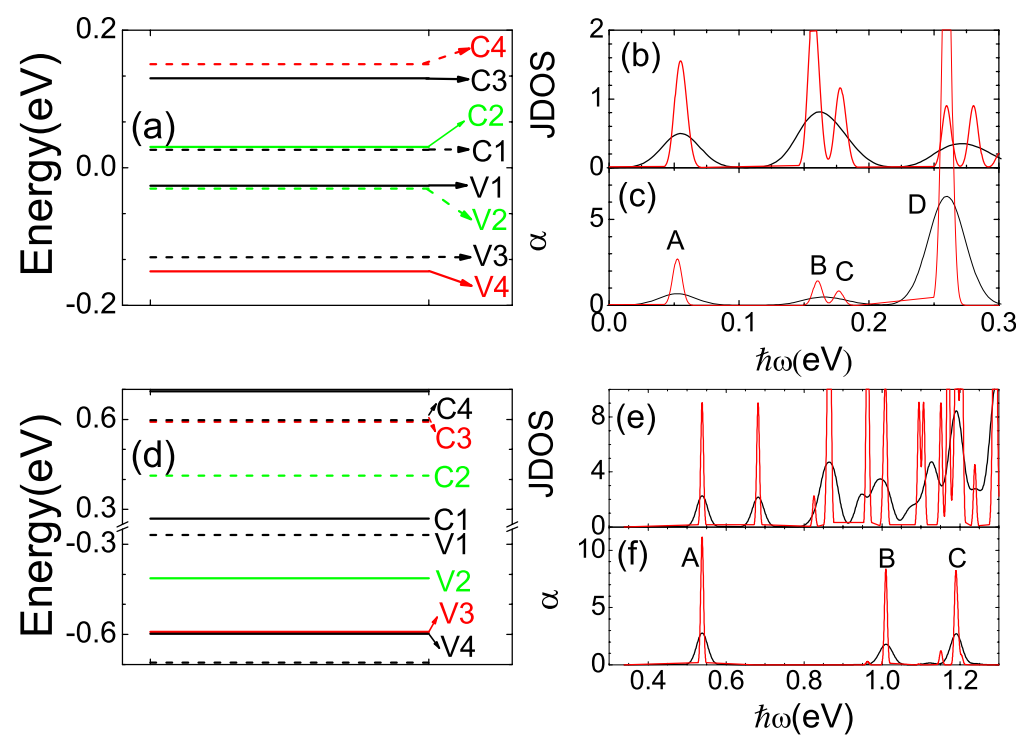

FIG. 6. (Color online) (a) and (d) are the level diagram for $N_{z}=12 \mathrm{ZGQD}$ and $N_{a}=9$ AGQD without the magnetic field, where different symmetries are represented by different colors and lines: black solid, black dashed, red solid, red dashed, green solid, and green dashed lines for the $E_{1}, E_{2}, A_{1}, A_{2}, A_{3}$, and $A_{4}$ irreducible representations of the $C_{6 v}$ symmetry, respectively. (b) and (c) The jointed density of state (JDOS) and the optical-absorption spectrum $\alpha$ for $N_{z}=12$ ZGQD. We used a Gauss function with different broadening factors: 0.02 and $0.005 \mathrm{eV}$ for the black and red lines. (e) and (f) are the same as (b) and (c), but for the $N_{a}=9$ AGQD. with even $N_{z}$, the conduction band levels, from bottom to top, exhibit different symmetries, i.e., $E_{2}, A_{3}, E_{1}, A_{2}$, etc., and the corresponding valence band levels show $E_{1}, A_{4}, E_{2}$, $A_{1}$, etc. For the zigzag GQDs with odd $N_{z}$, the conduction band levels display the opposite (same) symmetries $E_{1}, A_{4}$, $E_{2}, A_{1}$, etc. to the conduction (valence) band levels of zigzag GQDs with even $N_{z}$. For the armchair GQDs, the lowest conduction band level always shows the symmetries $E_{1}, A_{4}$, $A_{2}, E_{2}$, etc. from bottom to top and this order is independent of the size $\left(N_{a}\right)$ of the armchair GQD.

For $N_{z}=12$ ZGQD, the lowest optical-absorption peak (peak A) corresponds to the transition between the lowest conduction band level $C_{1}$ with $E_{2}$ symmetry and the highest valence band level $V_{1}$ with $E_{1}$ symmetry. The second and third lowest transitions correspond to the transition between the level $C_{2}\left(C_{3}\right)$ with $A_{3}\left(E_{1}\right)$ symmetry and the level $V_{3}\left(V_{2}\right)$ with $E_{2}\left(A_{4}\right)$ symmetry and the level $C_{1}\left(C_{4}\right)$ and $V_{4}\left(V_{1}\right)$, respectively. But the strengths of these three transitions are very small, therefore these transitions are not clearly seen in the contour spectrum in Fig. 7 at $\Phi / \Phi_{0}=0$. The strong absorption peak (peak D) appears at $E=0.26 \mathrm{eV}$, corresponding to the transition between the level $C_{3}$ with $E_{1}$ symmetry and the level $V_{3}$ with $E_{2}$ symmetry. This strong absorption arises from the large moment matrix $\langle n|\mathbf{p}| m\rangle$ between these states.

TABLE I. Direct products of the $E_{2}$ representation for the momentum operator $p_{x}\left(p_{y}\right)$ with all the irreducible representations of the $C_{6 v}$ group. The results are presented as direct sums of all possible irreducible representations of the $C_{6} v$ group. The notations of symmetries are adopted from Ref. 23.

\begin{tabular}{lc}
\hline \hline Direct product & Direct sum \\
\hline$E_{2} \otimes A_{1}$ & $E_{2}$ \\
$E_{2} \otimes A_{2}$ & $E_{1}$ \\
$E_{2} \otimes A_{3}$ & $E_{2}$ \\
$E_{2} \otimes A_{4}$ & $E_{1}$ \\
$E_{2} \otimes E_{1}$ & $A_{2} \oplus A_{4} \oplus E_{2}$ \\
$E_{2} \otimes E_{2}$ & $A_{1} \oplus A_{3} \oplus E_{1}$ \\
\hline \hline
\end{tabular}

For $N_{a}=9 \mathrm{AGQD}$, the lowest peak (peak A) is similar to that in the zigzag GQD, corresponding to the transition between $C_{1}$ and $V_{1}$. But the second peak (peak B) is different from those of the zigzag GQD. This peak corresponds to the transition between the level $C_{2}\left(C_{4}\right)$ with $A_{4}\left(E_{2}\right)$ symmetry and the level $V_{4}\left(V_{2}\right)$ with $E_{1}\left(A_{3}\right)$ symmetry. The third strong peak (peak $\mathrm{C}$ ) indicates the transition between the level $C_{4}$ with $E_{2}$ symmetry and the level $V_{4}$ with $E_{1}$ symmetry. Strong absorption takes place when the initial $\left(V_{i}\right)$ and final states $\left(C_{i}\right)$ have either $E_{1}$ or $E_{2}$ symmetry. As the size of the GQD increases, the absorption peaks shift to a long wavelength for both ZGQD and AGQD. The absorption peaks of the ZGQD shift to the long wavelength faster than those of the AGQD. The relative strength between the peak $D$ and $A$ increases as the size of the GQD increases for ZGQDs. But for AGQDs, the relative strength between the peak $C$ and $A$ is almost independent of the size.

Next, we discuss the effect of a magnetic field on the optical spectrum of a GQD. Here, we only focus on the small magnetic flux case (see Fig. 7). The spectra of two distinct GQDs, the zigzag and armchair GQDs, exhibit quite a different behavior due to their different level structures and the oscillator strengths determined by the boundary especially for the LCL and HVL, which localize at the edge of ZGQD. The spectra of the two distinct GQDs show that the strengths of the transitions vary as the magnetic field increases. In

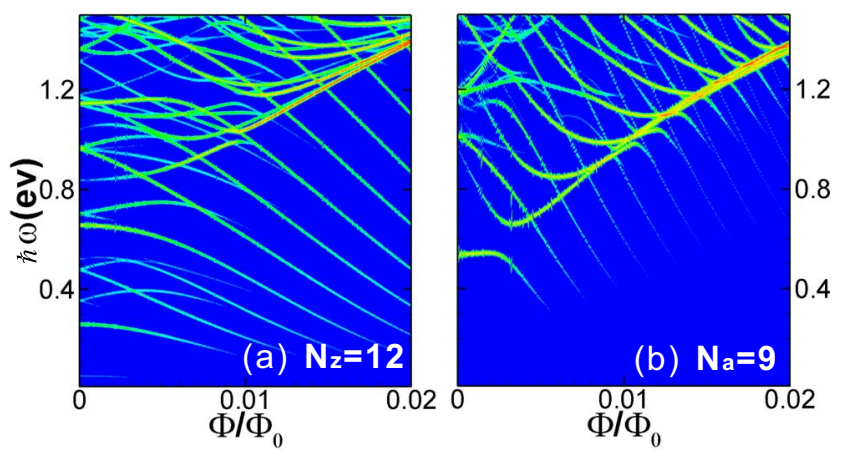

FIG. 7. (Color online) The contour plot of the magneto-optical spectra of (a) the zigzag and (b) the armchair GQD, respectively. 
particular, the strong absorption lines exhibit $\sqrt{B}$ asymptotic behavior corresponding to the transitions between the conduction and valence band Landau levels at a high magnetic field. We also find anticrossings in the spectra since the magnetic field induces the mixing of the levels belonging to the different families.

In summary, we investigated theoretically the magnetic levels and the optical spectrum in GQDs. In contrast to conventional semiconductor QDs, the LCL and HVL exhibit an edge-state feature, i.e., a nonzero probability of being at the edge of the sample, and the density distribution depends sensitively on the type of boundary of GQDs and the magnetic field strength. The magnetic levels of GQD display a Hofstadter-butterfly characteristic and approach the Landau levels of two-dimensional graphene as the magnetic field increases. The magneto-optical spectrum of a graphene quantum dot in the interesting energy range $(0-3 \mathrm{eV})$ is promising for carbon-based electronics applications. The position and strength of the absorption peaks can be tuned by the size of the GQD, the type of the edge of the GQD, and the external magnetic field.

This work is supported by the NSF of China Grant No. 60525405 and the Flander-China bilateral program.
*Corresponding author. kchang@red.semi.ac.cn

${ }^{1}$ K. S. Novoselov, A. K. Geim, S. V. Morozov, D. Jiang, Y. Zhang, S. V. Dubonos, I. V. Grigorieva, and A. A. Firsov, Science 306, 666 (2004); H. Hiura, Appl. Surf. Sci. 222, 374 (2004); Y. Zhang, J. W. Tan, H. L. Stormer, and P. Kim, Nature (London) 438, 201 (2005); A. K. Geim and K. S. Novoselov, Nat. Mater. 6, 183 (2007)

${ }^{2}$ K. S. Novoselov, Nature (London) 438, 197 (2005).

${ }^{3}$ P. G. Silvestrov and K. B. Efetov, Phys. Rev. Lett. 98, 016802 (2007).

${ }^{4}$ A. De Martino, L. Dell'Anna, and R. Egger, Phys. Rev. Lett. 98, 066802 (2007).

${ }^{5}$ H. Y. Chen, V. Apalkov, and T. Chakraborty, Phys. Rev. Lett. 98, 186803 (2007).

${ }^{6}$ J. M. Pereira, Jr., P. Vasilopoulos, and F. M. Peeters, Nano Lett. 7, 946 (2007).

${ }^{7}$ B. Trauzettel, D. V. Bulaev, D. Loss, and G. Burkard, Nat. Phys. 3, 192 (2007).

${ }^{8}$ T. G. Pedersen, C. Flindt, J. Pedersen, N. A. Mortensen, A.-P. Jauho, and K. Pedersen, Phys. Rev. Lett. 100, 136804 (2008).

${ }^{9}$ M. O. Goerbig, J. N. Fuchs, K. Kechedzhi, and V. I. Fal'ko, Phys. Rev. Lett. 99, 087402 (2007).

${ }^{10}$ V. P. Gusynin, S. G. Sharapov, and J. P. Carbotte, Phys. Rev. Lett. 98, 157402 (2007).
${ }^{11}$ H. Hsu and L. E. Reichl, Phys. Rev. B 76, 045418 (2007).

${ }^{12}$ B. Ozyilmaz, P. Jarillo-Herrero, D. Efetov, D. A. Abanin, L. S. Levitov, and P. Kim, Phys. Rev. Lett. 99, 166804 (2007).

${ }^{13}$ K. Nakada, M. Fujita, G. Dresselhaus, and M. S. Dresselhaus, Phys. Rev. B 54, 17954 (1996).

${ }^{14}$ K. Wakabayashi, M. Fujita, H. Ajiki, and M. Sigrist, Phys. Rev. B 59, 8271 (1999).

${ }^{15}$ R. Saito, G. Dresselhaus, and M. S. Dresselhaus, Physical Properties of Carbon Nanotubes (Imperial College, London, 1998).

${ }^{16}$ M. Ezawa, Phys. Rev. B 73, 045432 (2006).

${ }^{17}$ L. Brey and H. A. Fertig, Phys. Rev. B 73, 235411 (2006).

${ }^{18}$ T. Yamamoto, T. Noguchi, and K. Watanabe, Phys. Rev. B 74, 121409(R) (2006).

${ }^{19}$ D. R. Hofstadter, Phys. Rev. B 14, 2239 (1976); R. Rammal, J. Phys. (Paris) 46, 1345 (1985).

${ }^{20}$ Y. Hatsugai, T. Fukui, and H. Aoki, Phys. Rev. B 74, 205414 (2006).

${ }^{21}$ N. Nemec and G. Cuniberti, Phys. Rev. B 75, 201404(R) (2007).

${ }^{22}$ T. G. Pedersen, K. Pedersen, and T. B. Kriestensen, Phys. Rev. B 63, 201101(R) (2001).

${ }^{23}$ G. L. Bir and G. E. Pikus, Symmetry and Strain Induced Effects in Semiconductors (Wiley, New York, 1974); G. F. Koster, J. O. Dimmock, R. G. Wheeler, and H. Slatz, Properties of the ThirtyTwo Point Groups (MIT, Cambridge, 1966). 